\title{
Efficient Discrimination of Neutron and Gamma Rays using Different Digital Pulse Processing Algorithms
}

\author{
M. E. Hammad \\ Engineering Department, Nuclear \\ Research Center, Atomic Energy \\ Authority, Egypt \\ Sayed M. S. Elaraby \\ Dept. of Communications \\ Faculty of Electronic Engineeing, \\ Menofia University \\ Menouf, Egypt
}

\author{
H. Kasban \\ Engineering Department, Nuclear \\ Research Center, Atomic Energy \\ Authority, Egypt \\ M.I. Dessouky \\ Dept. of Communications \\ Faculty of Electronic Engineeing, \\ Menofia University \\ Menouf, Egypt \\ dr_moawad@yahoo.com
}

\author{
F. E. Abd El-Samie \\ Dept. of Communications \\ Faculty of Electronic Engineeing, \\ Menofia University \\ Menouf, Egypt
}

\author{
R. M. Fikry \\ Engineering Department, Nuclear \\ Research Center, Atomic Energy \\ Authority, Egypt \\ O. Zahran \\ Dept. of Communications \\ Faculty of Electronic Engineeing, \\ Menofia University \\ Menouf, Egypt
}

\begin{abstract}
In different neutron measurement experiments, it is necessary to apply pulse processing method to distinguish neutron pulses from gamma pulses. The discrimination process is based on the different decaying response of the detector for both neutron and gamma events. Different proposed algorithms are presented in this paper for determining the radiation type of detector output. In the proposed algorithms, features are extracted from the input radiation event. These features are extracted using charge integration, Hilbert transformation, and matched filtering methods. The extracted features are then fed the discriminator which is an Artificial Neural Network (ANN) or Support Vector Machine (SVM) discriminators. The obtained results prove that, the proposed approaches can be used efficiently for the neutron and gamma discrimination purpose and that method based on the Hilbert transformation achieves the highest discrimination rates.
\end{abstract}

Keywords: Neutron and Gamma Rays Discrimination, PSD, SVM, ANN

\section{Introduction}

Scintillators are used for the detection purpose of neutron radiation from an accelerator or from a research reactor in the presence of a high degree of gamma background. Therefore, for nuclear physics applications, discriminating neutron events from the background gamma ones is a very vital process. It based on the fact that gammas are detected with faster scintillation response compared to neutrons as shown in Fig. 1. The traditional Pulse Shape Discrimination (PSD) includes the two widely used methods of rising time of the detector output events. The time intervals between two different amplitudes of the pulse is measured and used for the first method [1]. The comparison of relative amount of emitted light during its slow and fast components is the basis for the other method [2].

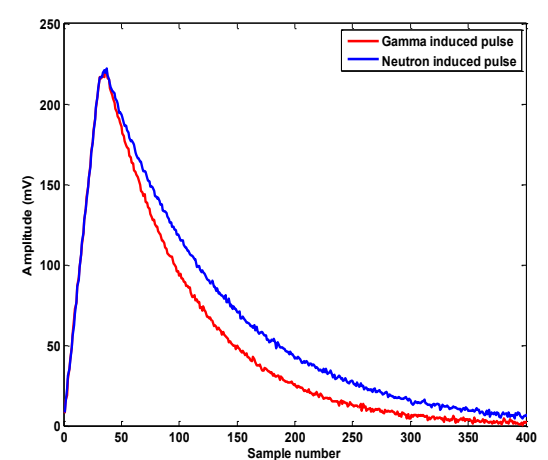

Figure (1): Decay from neutron and gamma induced scintillation pulses.

The traditional analogue PSD has been replaced by the digital PSD due to the availability of high-speed waveform digitizers. Therefore, complicated data analysis that are not accessible in analogue systems can be acquired. For example, S. D. Jastaniah et al. [3] used the digital pulse rise time to achieve good $\mathrm{n} / \gamma$ discrimination in boron loaded liquid scintillator. In 2014, Abdullah Al-Shbatat [4] used different numerical methods like trapezoidal, Simpson's, and rectangular integration methods pulse integration and used its value for the discrimination. Simpson's and Trapezoidal results reveal superiority for PSD although they didn't give completely discrimination at low energies.

Recently, Chuan et al. [5] presented four neutrongamma discrimination methods with EJ-301 scintillator. The four methods are Charge Comparison (CC), threshold Crossing Time (TCT), Correlation Pattern Recognition (CPR), and Pulsed Gradient Analysis (PGA) methods. The $\mathrm{CPR}$ and $\mathrm{CC}$ methods have good discrimination but limited at low energy threshold. The CC strongly depends on linearity and deformation of the pulse tail. Qiao et al. [6] used the convolution neural networks to discriminate double 
beta decay signal and background from high energy gammas.

Liang et al. discus the effect of the temperature variation on the PSD result for a designed array of Silicon Photo-Multiplier (SiPM) coupled to Lithium-loaded scintillators. Results indicated that a poorer discrimination is obtained at high temperatures because of the thermal noise in SiPMs [7]. Yang et al. investigated and compared four FPGA-based digital PSD methods. The included methods are CC, Pulse Peak Analysis (PPA), PGA, and finite impulse response Filter Comparison Method (FCM) [8]. Yang et.al [9] incorporated Li into the matrix of $\mathrm{NaI}$ under an optimized crystal growth process for efficient neutron detection. They demonstrated the use of exceptional gamma-neutron PSD.

This paper presents different proposed algorithms for the PSD process of neutron and gamma pulses by applying different signal processing techniques. The reset of the paper is organized as follow; Section 2 presents the traditional method for PSD. Section 3 presents the different algorithms that proposed for PSD. Simulation results and the comparison between the traditional and proposed method are illustrated in Section 4

\section{Traditional PSD based on Charge-Integration Method}

The traditional technique is based on using a chargeintegrating Analogue to Digital Converter (ADC) and two windows with different size [2]; one to integrate the entire charge of the pulse, and the other to integrate its tail component. This method takes advantage of the difference in time distribution between neutron and gamma pulses. The selected size of the windows is based upon the measurement setup and can differ from one setup to another. Similar techniques have been proposed by researchers [10-17]. These methods are selected to be consistent with the expected time distribution for a neutron or a gamma.

The integral ratios $\mathrm{R}$ of Eq. 1 for the two pulse intervals, one covering the tail and the other covering the whole pulse have been calculated [11]. These two intervals are shown in Fig. 2 where $T_{1}$ is the starting point of the total integral $\left(A_{1}\right)$, $\mathrm{T}_{2}$ is the starting point of the tail integral $\left(\mathrm{A}_{2}\right)$ and $\mathrm{T}_{\text {end }}$ is the ending point of both. Since neutron pulses have a larger fraction of light in the tail, a larger ratio $\mathrm{R}$ will be obtained for neutrons compared to $\gamma$-rays. The estimated value $\mathrm{R}$ is used accordingly as a discriminating feature that used to distinguish neutron pulses from $\gamma$-ray pulses.

$$
\mathrm{R}=\frac{\text { tail integral }}{\text { total integral }}=\frac{\mathrm{A}_{2}}{\mathrm{~A}_{1}}
$$

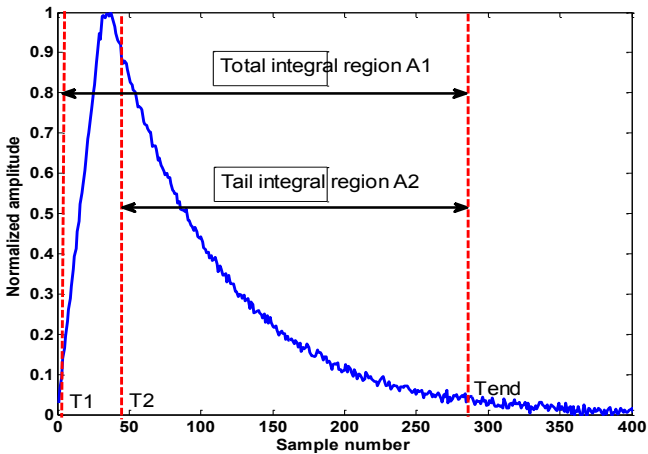

Fig. 2: Tail and total pulse integral regions for traditional charge-integration method

\section{Proposed PSD Methods}

PSD process is based on the fact that neutron and gamma produce light scintillations with significantly different decay characteristics. A general proposed technique based on using different tools of signal processing for extracting features consists of two phases; a training phase and a testing phase as configured in Fig. 3. The extracted features are used to build a database model for each signal type during the training phase. The radiation type of the applied pulse is determined during the test phase using a feature matching process.

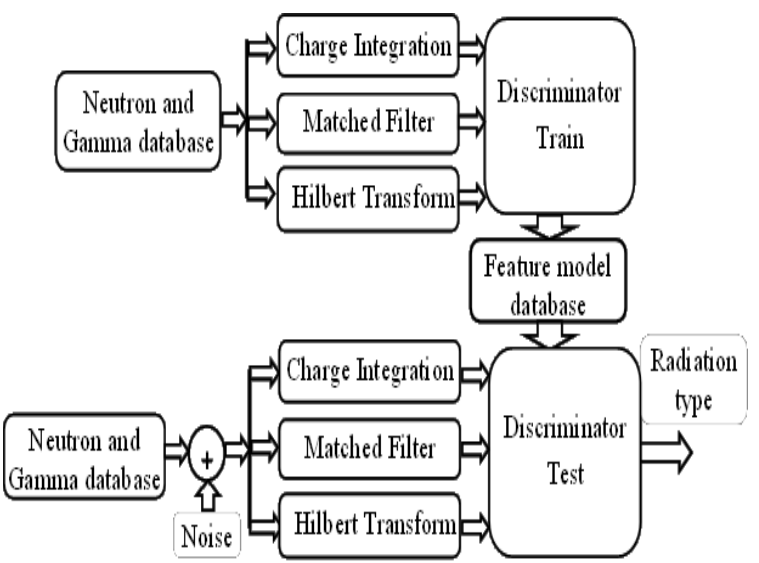

Fig. 3: General block diagram of the proposed algorithms for PSD

\subsection{Data Description}

The proposed PSD methods are evaluated using simulated input signals generated using MATLAB environment. The mathematical fitting model presented in $[18,19,20]$ is used for these generation. To observe the ability of the proposed approaches at lower and higher energies, the height of the simulated pulse is chosen to cover these energy bands $[11,12]$. The generated signals are assumed to be digitized with $0.1 \mathrm{~ns}$ sampling time.

The applied database consists of 3500 gamma events and 2900 neutron events with different energies. The different discriminator of the proposed and the traditional algorithms are trained using $40 \%$ of the input database of neutron and gamma pulses. The training pattern are selected to include the different energy band of the neutron and gamma radiation pulses. Also the discriminators of all algorithms 
are tested with the whole database of input gamma and neutron pulses contaminated with Additive White Gaussian Noise (AWGN) with different values of Signal-to-Noise Ratio (SNR). The effect of pulse height variation on the performance of the proposed algorithms is studied by normalizing the applied input data using Eq. (2) as shown in Fig. 4, where $x(n), \min (x(n))$, and $\max (x(n))$ are respectively the applied radiation event, its minimum and maximum values. The proposed algorithm is then applied separately to both the direct and the normalized database.

$$
\mathrm{x}(\mathrm{n})=\frac{\mathrm{x}(\mathrm{n})-\min [\mathrm{x}(\mathrm{n}))}{[\max (\mathrm{x}(\mathrm{n}))-\min [(\mathrm{x}))]}
$$

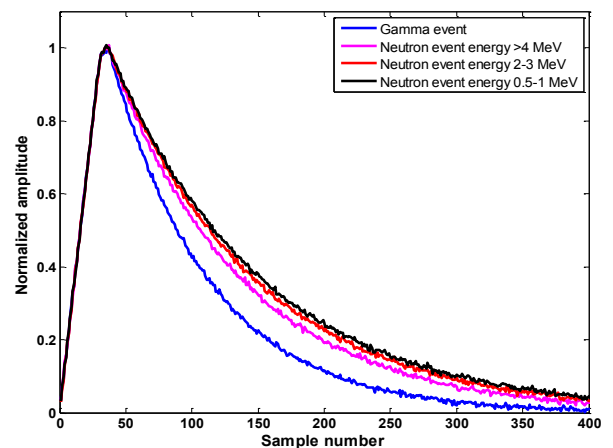

Fig. 4: Normalized plot of neutron and gamma induced scintillation pulses

\subsection{Features Extraction}

The process of feature extraction process is performed using different tools of signal processing in both phases. This paper discusses the ability of these tools to reduce the effect of noise in order to increase the discrimination rates.

\subsubsection{Feature Extraction using Matched Filter}

Matched filter is used frequently at receiver stations and is considered as a linear filter designed with the ability to detect the presence of waveform with known structure buried in noise. It hence maximizes the SNR of the detected signal as it responds by a sharp peak for the existence of the desired input pulse. The impulse response $\mathrm{h}(\mathrm{t})$ and frequency response $H(\omega)$ of the matched filter is deduced using the configured system of Fig.5.

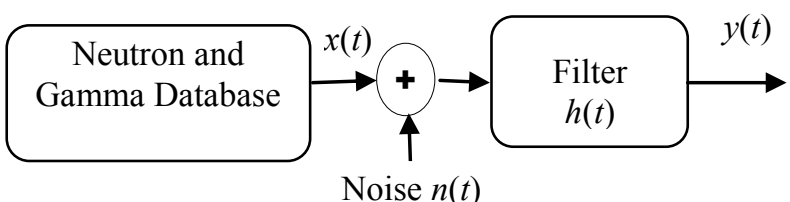

Fig. 5: Basic matched filter.

Matched filter of this system is represented as linear time invariant (LTI) system with input corrupted with noise $n(t)$. Accordingly, the system output consists of a signal component $y(t)$ and a noise component $n_{o}(t)$ which can be estimated as [21]:

$$
\begin{array}{r}
y(n)=x(n) \circledast h(n)=\sum_{k=0}^{N-1} X(k) H(k) e^{i \frac{2 \pi}{N} k n}, \quad n= \\
0,1, \ldots, N-1
\end{array}
$$

$$
\begin{array}{r}
\mathrm{n}_{\mathrm{o}}(\mathrm{n})=\mathrm{n}(\mathrm{n}) \circledast \mathrm{h}(\mathrm{n})=\sum_{\mathrm{n}=0}^{\mathrm{N}-1} \mathrm{~N}(\mathrm{k}) \mathrm{H}(\mathrm{k}) \mathrm{e}^{\mathrm{i} \frac{2 \pi}{\mathrm{N}} \mathrm{kn}}, \mathrm{n}= \\
0,1, \ldots, \mathrm{N}-1
\end{array}
$$

Equation (5) represents signal power component of matched filter output expressed in terms of its amplitude at sample $\mathrm{n}_{\mathrm{d}}$. The average noise power at the output of LTI system also can be estimated as in Eq. 6 in terms of spectral density $\mathrm{S}_{\mathrm{n}}(\mathrm{k})$ of the added noise as [21]:

$$
\begin{aligned}
& \left|\mathrm{y}\left(\mathrm{n}_{\mathrm{d}}\right)\right|^{2}=\left|\sum_{\mathrm{k}=0}^{\mathrm{N}-1} \mathrm{X}(\mathrm{k}) \mathrm{H}(\mathrm{k}) \mathrm{e}^{\mathrm{i} \frac{2 \pi}{\mathrm{N}} \mathrm{kn}} \mathrm{d}\right|^{2} \\
& \left|\mathrm{n}_{\mathrm{o}}(\mathrm{n})\right|^{2}=\sum_{\mathrm{k}=0}^{\mathrm{N}-1} \mathrm{~S}_{\mathrm{n}}(\mathrm{k})|\mathrm{H}(\mathrm{k})|^{2}
\end{aligned}
$$

The SNR at the matched filter output is then can be estimated as [21]:

$$
\operatorname{SNR}=\frac{\left|\mathrm{y}\left(\mathrm{n}_{\mathrm{d}}\right)\right|^{2}}{\left|\mathrm{n}_{\mathrm{o}}(\mathrm{n})\right|^{2}}=\frac{\left|\sum_{\mathrm{k}=0}^{\mathrm{N}-1} \mathrm{X}(\mathrm{k}) \mathrm{H}(\mathrm{k}) \mathrm{e}^{\mathrm{i} \frac{2 \pi}{\mathrm{N}} \mathrm{kn}} \mathrm{d}\right|^{2}}{\sum_{\mathrm{k}=0}^{\mathrm{N}-1} \mathrm{~S}_{\mathrm{n}}(\mathrm{k})|\mathrm{H}(\mathrm{k})|^{2}}
$$

Making use of a Schwartz inequality relationship and with some simplifications, the maximum SNR at time nd can be obtained when the system frequency response satisfies [21]:

$$
H(k)=C \cdot \frac{X^{*}(k)}{S_{n}(k)} e^{-i \frac{2 \pi}{N} k n_{d}}, k=0,1, \ldots, N-1
$$

Where $\mathrm{C}$ is a real constant, usually positive and often set to unity. For a white noise with zero mean, $S_{n}(k)$ is constant and hence the filter impulse and frequency response becomes [21]:

$$
\begin{aligned}
& H(k)=C \cdot X^{*}(\omega) e^{-j \omega t_{d}} \\
& h(n)=C x^{*}\left(n_{d}-n\right)
\end{aligned}
$$

The final result indicates that the matched filter impulse response that enhances SNR is the time reversal of its input signal. Therefore optimum filter of the proposed PSD algorithm will have two forms to reduce the effect of noise in the discrimination process. The first one is the time reversal of a gamma pulse and the other is the time reversal of a neutron pulse. The different matched filter output is then used by the discriminator for the discrimination process.

\subsubsection{Discrete Time Analytic Signal using Hilbert}

\section{Transform}

In signal processing, the Hilbert transform is a specific linear operator that takes a real function, $\mathrm{x}(\mathrm{t})$ and produces another function of $\mathrm{H}(\mathrm{x}(\mathrm{t}))$. This linear operator is a $( \pm \pi / 2)$ phase-shift as the German scientist David Hilbert indicates. He showed that the function $\sin (\omega n)$ is the Hilbert transform of $\cos (\omega n)$. The Hilbert transform shown in Eq. 11 can be thought as the convolution of $x(n)$ with the Hilbert transformer $h(n)=1 /(\pi n)$, known as the Cauchy kernel.

$$
\begin{array}{r}
\mathrm{H}(\mathrm{x}(\mathrm{n}))=\sum_{\mathrm{k}=0}^{\mathrm{N}-1} \mathrm{x}(\mathrm{k}) \mathrm{h}(\mathrm{n}-\mathrm{k})=\frac{1}{\pi} \sum_{\mathrm{k}=\mathrm{o}}^{\mathrm{N}-1} \frac{\mathrm{x}(\mathrm{k})}{\mathrm{n}-\mathrm{k}}, \\
\mathrm{n}=0,1, \ldots, \mathrm{N}-1
\end{array}
$$

A real function $\mathrm{x}(\mathrm{n})$ and its Hilbert transform are related to each other in such a way that they together create a so called 
strong analytic signal which can be written with amplitude and phase components. The analytic signal for a sequence has a one-sided Fourier transform. To get an approximate estimate of the analytic signal, Hilbert calculates the FFT of the input sequence. The coefficients of negative frequencies are then replaced with zeros. Finally, the approximate is the inverse FFT. It is a simple algorithm for instantaneous frequency extraction of a signal. Also, Hilbert transform is useful for envelope detection of a signal.

\subsection{Discriminator}

Discriminator is the final stage in both phases of the PSD algorithm that uses the extracted features using the different tools to classify the applied inputs to their types. Two discriminators are considered for this work, ANN and SVM [22]. To determine which of them is suitable for discrimination, a comparison between the obtained results is performed.

\subsubsection{Discrimination using Artificial Neural Networks.}

A Multi-Layer Perceptron (MLP) neural network is used for feature matching process. It consists of three layers; input, hidden and output layers. The input layer has an input vector $\mathrm{X}$ which is a vector of the discriminating features with size differs based on the used signal processing tool. The used hidden layer of the ANN has 125 neurons. The output vector is a vector containing an output for each signal type, so the output vector in this approach consists of two outputs. The network weights are adjusted during training by minimizing the sum of the squared error between the desired output $D_{0}$ and the actual output $\mathrm{Y}_{\mathrm{o}}$ given by $[23,24]$ :

$$
\mathrm{E}=\frac{1}{2} \sum_{\mathrm{o}=1}^{0}\left(\mathrm{D}_{\mathrm{o}}-\mathrm{Y}_{\mathrm{o}}\right)^{2}
$$

Where, $\mathrm{O}$ is the number of neurons in the output layer. The network weights that have acceptable value of $E$ are used to build a database model for each radiation type. During testing phase, features are extracted from a noisy database using the different estimation tools, then a feature matching between the extracted features and the saved database models is performed. The last stage in the recognition system is the decision making that takes the matching score as its input and makes the final decision about the type of radiation.

\subsubsection{Discrimination using Support Vector Machine.}

SVMs are a set of related algorithms of supervised learning used for discrimination and regression [25]. They are considered one of the generalized linear identification families. SVMs are characterized by a special merit where they achieve maximum margin with maintaining a minimized error of identification. So SVMs are called maximum margin discriminators. Maximal separating hyperplane is constructed by mapping an input vector to a higher dimensional space. On each side of the hyperplane that separates the data, there will be a parallel hyperplane. The separating hyperplane is the one that maximizes the distance between the two parallel hyperplanes. SVs are those training points which laying on one of the two hyperplanes, and whose removal would change the solution found [25].

\section{Results and Discussion}

MATLAB simulation environment has been used for the verification of this proposed PSD algorithms. Discrimination rate and error have been used as a performance metric for the discrimination methods. The discrimination rate is mathematically described by Eq. 13 which represents the ability of the method to discriminate the signals correctly even in high noisy environments.

\section{Discrimination rate $=$}

$$
\frac{\text { Number of success discrimination }}{\text { total number of discrimination trials }} * 100
$$

Another metric called discrimination error is used to clarify the ability of the proposed methods to discriminate one type of the signals while it failed to discriminate the other type. The performance of the proposed algorithms is studied by adding AWGN to the database with different SNRs. The following sections illustrate results of applying the traditional and the proposed PSD methods to direct and normalized signals separately using ANN and SVM.

\subsection{ANN Discriminator Results}

In this section, features from a noisy database have been extracted and applied to an ANN of an input layer with a different size based on the discrimination method, a hidden layer of 125 neurons, and an output layer of two neurons. The discrimination has been performed using both direct and normalized noisy database.

The discrimination results obtained using the traditional method with ANN for both direct and normalized database is shown in Table (1). The normalization process as indicated disturbs the obtained results largely as it scales the area under the pulse by a factor equal to the difference between its maximum and minimum values resulting in a reduction of the area difference between pulses. Accordingly, the discrimination is degraded since the main factor of the discrimination is this area value. The indicated results confirm the ability of the method in discriminating gamma signals with a very small percentage of error in case of applying the method on the direct signal. But, it fails in identifying neutron pulses with the same small percent of error.

The discrimination result obtained from using a matched filter with a transfer function equals to the time reversal of a radiation gamma pulse is indicated in Table 2 . It shows a slight enhancement in the discrimination rates compared to the traditional method results of table (1) for both direct and normalized input pulses. Also it confirms the same conclusion where the process of normalizing the radiation pulses before applying the PSD method, negatively affect the discrimination results. The obtained results for the case of pulse normalization as shown did not improved until a value of SNR equals to $25 \mathrm{~dB}$. Comparing the results of table (1) with table (2) indicates that, applying matched filter for PSD reach a full percentage of identification at 15 $\mathrm{dB}$ compared to $20 \mathrm{~dB}$ for the traditional charge integration method. So, matched filter with this impulse response slightly improves the discrimination process. 
Table (1): Discrimination results using traditional method with ANN

\begin{tabular}{|c|c|c|c|c|c|c|}
\hline \multirow{3}{*}{$\begin{array}{l}\text { SNR } \\
(\mathrm{dB})\end{array}$} & \multirow{2}{*}{\multicolumn{2}{|c|}{$\begin{array}{c}\text { Discrimination rate } \\
(\%)\end{array}$}} & \multicolumn{4}{|c|}{ Discrimination error $(\%)$} \\
\hline & & & \multicolumn{2}{|c|}{$\begin{array}{c}\text { Gamma } \\
\text { misclassification }\end{array}$} & \multicolumn{2}{|c|}{ Neutron misclassification } \\
\hline & $\begin{array}{l}\text { Direct } \\
\text { signal }\end{array}$ & $\begin{array}{l}\text { Normalized } \\
\text { signal }\end{array}$ & $\begin{array}{l}\text { Direct } \\
\text { signal }\end{array}$ & $\begin{array}{c}\text { Normalized } \\
\text { signal }\end{array}$ & $\begin{array}{l}\text { Direct } \\
\text { signal }\end{array}$ & $\begin{array}{c}\text { Normalized } \\
\text { signal }\end{array}$ \\
\hline-20 & 51.1364 & 49.7253 & 8.4291 & 25.0624 & 40.4346 & 25.2123 \\
\hline-15 & 52.4600 & 50.6618 & 8.2667 & 24.7003 & 39.2732 & 24.6379 \\
\hline-10 & 55.7443 & 50.4121 & 7.4301 & 24.6628 & 36.8257 & 24.9251 \\
\hline-5 & 59.9401 & 51.0365 & 5.6818 & 24.6004 & 34.3781 & 24.3631 \\
\hline 0 & 68.8811 & 51.5609 & 3.9336 & 24.1758 & 27.1853 & 24.2632 \\
\hline 5 & 82.8172 & 51.9481 & 2.1354 & 23.3267 & 15.0475 & 24.7253 \\
\hline 10 & 95.8916 & 53.9835 & 0.2997 & 23.1893 & 3.8087 & 22.8272 \\
\hline 15 & 99.7378 & 55.2448 & 0.0125 & 22.8646 & 0.2498 & 21.8906 \\
\hline 20 & 100 & 59.0160 & 0 & 21.7782 & 0 & 19.2058 \\
\hline 25 & 100 & 66.7582 & 0 & 19.0435 & 0 & 14.1983 \\
\hline 30 & 100 & 77.1104 & 0 & 15.0724 & 0 & 7.8172 \\
\hline 35 & 100 & 89.3232 & 0 & 8.3541 & 0 & 2.3227 \\
\hline 40 & 100 & 97.4525 & 0 & 2.3976 & 0 & 0.1499 \\
\hline 45 & 100 & 99.9001 & 0 & 0.0999 & 0 & 0 \\
\hline 50 & 100 & 100 & 0 & 0 & 0 & 0 \\
\hline
\end{tabular}

Table (2): Discrimination results using gamma based matched filter with ANN

\begin{tabular}{|c|c|c|c|c|c|c|}
\hline \multirow{3}{*}{$\begin{array}{l}\text { SNR } \\
\text { (dB) }\end{array}$} & \multirow{2}{*}{\multicolumn{2}{|c|}{$\begin{array}{c}\text { Discrimination Rate } \\
(\%)\end{array}$}} & \multicolumn{4}{|c|}{ Discrimination error $(\%)$} \\
\hline & & & \multicolumn{2}{|c|}{$\begin{array}{c}\text { Gamma } \\
\text { misclassification }\end{array}$} & \multicolumn{2}{|c|}{$\begin{array}{c}\text { Neutron } \\
\text { misclassification }\end{array}$} \\
\hline & $\begin{array}{l}\text { Direct } \\
\text { signal }\end{array}$ & $\begin{array}{c}\text { Normalized } \\
\text { signal }\end{array}$ & $\begin{array}{l}\text { Direct } \\
\text { signal }\end{array}$ & $\begin{array}{c}\text { Normalized } \\
\text { signal }\end{array}$ & $\begin{array}{l}\text { Direct } \\
\text { signal }\end{array}$ & $\begin{array}{l}\text { Normalized } \\
\text { signal }\end{array}$ \\
\hline-20 & 54.3457 & 50.1124 & 19.1434 & 0.8242 & 26.5110 & 49.0634 \\
\hline-15 & 55.8941 & 50.1249 & 17.9695 & 0.9615 & 6.1364 & 48.9136 \\
\hline-10 & 61.0639 & 50.1499 & 15.8716 & 0.8367 & 3.0644 & 49.0135 \\
\hline-5 & 68.5814 & 50.3996 & 13.6614 & 0.6993 & 7.7572 & 48.9011 \\
\hline 0 & 79.2458 & 50.4745 & 9.1034 & 0.7867 & 11.6508 & 48.7388 \\
\hline 5 & 91.9580 & 50.8492 & 3.0969 & 0.6369 & 4.9451 & 48.5140 \\
\hline 10 & 99.1508 & 51.5984 & 0.2248 & 0.5495 & 0.6244 & 47.8521 \\
\hline 15 & 100 & 52.4975 & 0 & 0.4870 & 0 & 47.0155 \\
\hline 20 & 100 & 55.8816 & 0 & 0.2248 & 0 & 43.8936 \\
\hline 25 & 100 & 3.0120 & 0 & 0.0874 & 0 & 36.9006 \\
\hline 30 & 100 & 74.6628 & 0 & 0.0250 & 0 & 25.3122 \\
\hline 35 & 100 & 90.5470 & 0 & 0 & 0 & 9.4530 \\
\hline 40 & 100 & 99.1384 & 0 & 0 & 0 & 0.8616 \\
\hline 45 & 100 & 100 & 0 & 0 & 0 & 0 \\
\hline
\end{tabular}

Table (3): Discrimination results using Neutron based matched filter with ANN

\begin{tabular}{|c|c|c|c|c|c|c|}
\hline \multirow{2}{*}{$\begin{array}{l}\text { SNR } \\
(\mathbf{d B})\end{array}$} & \multirow{2}{*}{ Discrimination Rate (\%) } & \multicolumn{3}{|c|}{$\begin{array}{c}\text { Giscrimination error (\%) } \\
\text { misclassification }\end{array}$} & $\begin{array}{c}\text { Neutron } \\
\text { misclassification }\end{array}$ \\
\cline { 2 - 7 } & $\begin{array}{c}\text { Direct } \\
\text { signal }\end{array}$ & $\begin{array}{c}\text { Normalized } \\
\text { signal }\end{array}$ & $\begin{array}{c}\text { Direct } \\
\text { signal }\end{array}$ & $\begin{array}{c}\text { Normalized } \\
\text { signal }\end{array}$ & $\begin{array}{c}\text { Direct } \\
\text { signal }\end{array}$ & $\begin{array}{c}\text { Normalized } \\
\text { signal }\end{array}$ \\
\hline-20 & 53.0220 & 52.2353 & 21.0415 & 25.7617 & 5.9366 & 22.0030 \\
\hline-15 & 54.3831 & 52.4725 & 20.7542 & 25.8991 & 4.8626 & 21.6284 \\
\hline-10 & 57.0554 & 56.7807 & 19.2682 & 23.1893 & 3.6763 & 20.0300 \\
\hline-5 & 63.4740 & 61.0265 & 16.9955 & 20.6419 & 9.5305 & 18.3317 \\
\hline 0 & 73.2018 & 70.8042 & 12.0629 & 14.8477 & 4.7353 & 14.3482 \\
\hline 5 & 85.7393 & 82.0180 & 6.4935 & 8.4291 & 7.7672 & 9.5529 \\
\hline 10 & 96.6533 & 94.4306 & 1.2987 & 1.9106 & 2.0480 & 3.6588 \\
\hline 15 & 99.8501 & 99.5380 & 0.0125 & 0.050 & 0.1374 & 0.4121 \\
\hline 20 & 100 & 100 & 0 & 0 & 0 & 0 \\
\hline
\end{tabular}


Table (4): Discrimination results using Hilbert transformation with ANN discriminator

\begin{tabular}{|c|c|c|c|c|c|c|}
\hline \multirow{3}{*}{$\begin{array}{l}\text { SNR } \\
\text { (dB) }\end{array}$} & \multirow{2}{*}{\multicolumn{2}{|c|}{$\begin{array}{c}\text { Discrimination Rate } \\
(\%)\end{array}$}} & \multicolumn{4}{|c|}{ Discrimination error $(\%)$} \\
\hline & & & \multicolumn{2}{|c|}{$\begin{array}{c}\text { Gamma } \\
\text { misclassification }\end{array}$} & \multicolumn{2}{|c|}{$\begin{array}{c}\text { Neutron } \\
\text { misclassification }\end{array}$} \\
\hline & $\begin{array}{l}\text { Direct } \\
\text { signal }\end{array}$ & $\begin{array}{l}\text { Normalized } \\
\text { signal }\end{array}$ & $\begin{array}{l}\text { Direct } \\
\text { signal }\end{array}$ & $\begin{array}{c}\text { Normalized } \\
\text { signal }\end{array}$ & $\begin{array}{l}\text { Direct } \\
\text { signal }\end{array}$ & $\begin{array}{c}\text { Normalized } \\
\text { signal }\end{array}$ \\
\hline-20 & 56.2313 & 52.1229 & 22.4026 & 24.4880 & 21.3661 & 23.3891 \\
\hline-15 & 60.6269 & 54.1334 & 21.2662 & 23.2892 & 18.1069 & 22.5774 \\
\hline-10 & 69.2058 & 57.4301 & 17.2577 & 22.1903 & 13.5365 & 20.3796 \\
\hline-5 & 82.1928 & 62.8247 & 11.9755 & 19.9925 & 5.8317 & 17.1828 \\
\hline 0 & 93.2443 & 71.1913 & 5.6319 & 16.1963 & 1.1239 & 12.6124 \\
\hline 5 & 99.2507 & 82.5425 & 0.7368 & 11.1139 & 0.0125 & 6.3437 \\
\hline 10 & 0 & 95.5919 & 0 & 3.6588 & 0 & 0.7493 \\
\hline 15 & 0 & 99.7627 & 0 & 0.2373 & 0 & 0 \\
\hline 20 & 0 & 100 & 0 & 0 & 0 & 0 \\
\hline
\end{tabular}

Table (5): Discrimination results using traditional method with SVM discriminator

\begin{tabular}{|c|c|c|c|c|c|c|}
\hline \multirow{2}{*}{$\begin{array}{l}\text { SNR } \\
\text { (dB) }\end{array}$} & \multicolumn{2}{|c|}{$\begin{array}{c}\text { Discrimination Rate } \\
\text { (\%) }\end{array}$} & \multicolumn{4}{|c|}{ Discrimination error (\%) } \\
\cline { 2 - 7 } & $\begin{array}{c}\text { Direct } \\
\text { signal }\end{array}$ & $\begin{array}{c}\text { Normalized } \\
\text { signal }\end{array}$ & $\begin{array}{c}\text { Gamma } \\
\text { Direct } \\
\text { signal }\end{array}$ & $\begin{array}{c}\text { Normalized } \\
\text { signal }\end{array}$ & $\begin{array}{c}\text { Neutron } \\
\text { indiscrimination }\end{array}$ \\
\hline-20 & 57.2802 & 55.78172 & 21.229 & 22.85215 & 21.491 & 21.36613 \\
signal & $\begin{array}{c}\text { Normalized } \\
\text { signal }\end{array}$ \\
\hline-15 & 62.8871 & 59.62787 & 18.244 & 21.74076 & 18.8686 & 18.63137 \\
\hline-10 & 71.1663 & 66.13387 & 13.437 & 19.08092 & 15.3971 & 14.78521 \\
\hline-5 & 84.2158 & 75.28721 & 6.5934 & 15.85914 & 9.19081 & 8.85365 \\
\hline 0 & 96.1913 & 87.25025 & 1.2987 & 9.37812 & 2.50999 & 3.37163 \\
\hline 5 & 99.8626 & 95.54196 & 0.0125 & 3.97103 & 0.12488 & 0.48701 \\
\hline 10 & 100 & 99.13836 & 0 & 0.83666 & 0 & 0.02498 \\
\hline 15 & 100 & 99.93756 & 0 & 0.06244 & 0 & 0 \\
\hline 20 & 100 & 100 & 0 & 0 & 0 & 0 \\
\hline
\end{tabular}

Table (6): Discrimination results using gamma based matched filter with SVM

\begin{tabular}{|c|c|c|c|c|c|c|}
\hline \multirow{2}{*}{$\begin{array}{l}\text { SNR } \\
\text { (dB) }\end{array}$} & \multicolumn{2}{|c|}{$\begin{array}{c}\text { Discrimination Rate } \\
(\mathbf{\%})\end{array}$} & \multicolumn{3}{|c|}{ Discrimination error } \\
\cline { 4 - 7 } & $\mathbf{2} \%)$ & \multicolumn{2}{|c|}{$\begin{array}{c}\text { Gamma } \\
\text { misclassification }\end{array}$} & \multicolumn{2}{c|}{$\begin{array}{c}\text { Neutron } \\
\text { misclassification }\end{array}$} \\
\cline { 2 - 7 } & $\begin{array}{c}\text { Direct } \\
\text { signal }\end{array}$ & $\begin{array}{c}\text { Normalized } \\
\text { signal }\end{array}$ & $\begin{array}{c}\text { Direct } \\
\text { signal }\end{array}$ & $\begin{array}{c}\text { Normalized } \\
\text { signal }\end{array}$ & $\begin{array}{c}\text { Direct } \\
\text { signal }\end{array}$ & $\begin{array}{c}\text { Normalized } \\
\text { signal }\end{array}$ \\
\hline-20 & 63.14935 & 58.59141 & 19.543 & 19.85514 & 17.3077 & 21.55345 \\
\hline-15 & 71.09141 & 65.27223 & 16.084 & 16.12138 & 12.8247 & 18.60639 \\
\hline-10 & 83.52897 & 77.75974 & 10.527 & 10.15235 & 5.94406 & 12.08791 \\
\hline-5 & 93.84366 & 91.28372 & 4.8951 & 3.15934 & 1.26124 & 5.55694 \\
\hline 0 & 98.85115 & 98.81369 & 1.1114 & 0.13736 & 0.03746 & 1.04895 \\
\hline 5 & 99.92507 & 99.98751 & 0.0749 & 0 & 0 & 0.01249 \\
\hline 10 & 100 & 100 & 0 & 0 & 0 & 0 \\
\hline
\end{tabular}

Table (7): Discrimination results using Neutron based matched filter with SVM

\begin{tabular}{|c|c|c|c|c|c|c|}
\hline \multirow{3}{*}{$\begin{array}{l}\text { SNR } \\
\text { (dB) }\end{array}$} & \multirow{2}{*}{\multicolumn{2}{|c|}{$\begin{array}{c}\text { Discrimination Rate } \\
(\%)\end{array}$}} & \multicolumn{4}{|c|}{ Discrimination error $(\%)$} \\
\hline & & & \multicolumn{2}{|c|}{$\begin{array}{c}\text { Gamma } \\
\text { misclassification }\end{array}$} & \multicolumn{2}{|c|}{$\begin{array}{c}\text { Neutron } \\
\text { misclassification }\end{array}$} \\
\hline & $\begin{array}{l}\text { Direct } \\
\text { signal }\end{array}$ & $\begin{array}{l}\text { Normalized } \\
\text { signal }\end{array}$ & $\begin{array}{l}\text { Direct } \\
\text { signal }\end{array}$ & $\begin{array}{c}\text { Normalized } \\
\text { signal }\end{array}$ & $\begin{array}{l}\text { Direct } \\
\text { signal }\end{array}$ & $\begin{array}{c}\text { Normalized } \\
\text { signal }\end{array}$ \\
\hline-20 & 62.3626 & 59.17832 & 19.5430 & 21.07892 & 18.0944 & 19.74276 \\
\hline-15 & 72.1903 & 67.75724 & 16.0839 & 18.20679 & 11.7258 & 14.03596 \\
\hline-10 & 83.9036 & 76.09890 & 10.5270 & 14.81019 & 5.56943 & 9.09091 \\
\hline-5 & 94.0559 & 88.24925 & 4.8951 & 8.42907 & 1.04895 & 3.32168 \\
\hline 0 & 98.8137 & 95.62937 & 1.11139 & 3.85864 & 0.07493 & 0.51199 \\
\hline 5 & 99.9501 & 99.27572 & 0.07493 & 0.72428 & 0.04995 & 0 \\
\hline 10 & 100 & 99.95005 & 0 & 0.04995 & 0 & 0 \\
\hline 15 & 100 & 100 & 0 & 0 & 0 & 0 \\
\hline
\end{tabular}


Table (3) summarized the discrimination results obtained from using matched filter with a transfer function equals to the time reversal of radiation neutron pulse. It approximately did not improve the performance compared to results of table (2) for matched filter based on gamma pulse that has slightly better discrimination performance. The obtained results for a normalized database have been improved compared to table (1) and (2). However, slightly better results are still obtained when using direct signal. Generally, applying matched filter with a transfer function equals to the time reversal of a gamma radiation pulse on the direct database signals performs better.

Table (4) shows the discrimination results of applying Hilbert transform on both direct and normalized database. The performance as shown in this table has been improved compared to the charge integration and matched filter based methods. Hence, in case of using ANN discriminator, it is useful to apply this transformation for the PSD of neutron and gamma events especially when these events are detected at high noisy conditions.

\subsection{SVM Discriminator Results}

In this section, SVM with a linear kernel function has been used as a discriminator instead of the ANN discriminator. Tables (5) to (8) show the discrimination results using the same methods with SVM.

The obtained results of tables (5) to (8) reveal the same conclusion where applying the normalization process didn't enhance the discrimination performance as the case when using direct not normalized inputs. Although the process of normalizing input data reduce the effect of the different energy value of the applied signals, it also increases the similarity between them and hence minimizes the discrimination rate.

It is clear from the obtained results that, applying Hilbert transformation succeeded efficiently for discriminating the type of the input radiation. The other signal processing tools can be used also for this purpose, but with slightly larger discrimination errors. The SVM discriminator results agree with the same approved idea by ANN results that, normalizing the input database before applying the discrimination process does not improve the performance of the traditional and the matched filter based discrimination methods. Also, to decide which type of discriminators gives the highest discrimination rates, the obtained results using SVMs as discriminator are compared with that obtained using ANNs.
The comparison proved the superiority of SVM discriminator as they achieved better performance than the ANNs discriminator at the same noise level. Utilizing the SVM discriminator achieves another advantage as it needs slightly shorter discrimination times than the ANN discriminator. Accordingly, it is preferable for good PSD to apply the Hilbert based method on the input database with the use of the SVMs discriminator.

\section{References}

[1] G. Ranucci, "An Analytical Approach To The Evaluation Of The Pulse Shape Discrimination Properties Of Scintillators", Nucl. Instr. Methods, Vol. A354, pp. 389-399, 1995.

[2] J. H. Heltsley, L. Brandon, A. Galonsky, L. Heilbraun, B. A. Remington, S. Langer, A. Vander and J. Yourkon, "Particle Identification Via Pulse Shape Discrimination With A ChargeIntegrating $A D C^{\prime}$ ', Nuclear Inst. and Methods in Physics Research Section A: Accelerators, Spectrometers, Detectors and Associated Equipment, Vol. A263, pp. 441-445, 1988.

[3] P. J. Sellin, G. Jaffar and S. D. Jastaniah, "Performance of Digital Algorithms for $n / \gamma$ Pulse Shape Discrimination using A Liquid Scintillation Detector", IEEE, Vol. 2, pp. 1057-1060, 2004.

[4] A. Al-Shbatat, "Analysis of the Spectrum of Radiation Detectors", Msc thesis, Faculty of Informatics Sciences, Masaryk University, Brno, spring 2014

[5] P. V, Chuan and N. X. Hai, "Evaluating Four Neutron-Gamma Discrimination Methods With Ej-301 Scintillator", Analog Integrated Circuits and Signal Processing, Vol. 98, No. 1, pp. 1-10, 2018.

[6] H. Qiao, C. Y. Lu, X. Chen, K. Han, X. D. Ji, and S. Wang, "SignalBackground Discrimination With Convolutional Neural Networks In The Panda X-III Experiment using Mc Simulation", Science China Physics, Mechanics \& Astronomy, Vol. 61, pp. 101007-1101007-9, 2018

[7] F. Liang, H. Brands, L. Hoy, J. Preston, and J. Smith, "LithiumLoaded Scintillators Coupled To A Custom-Designed Silicon Photomultiplier Array For Neutron And Gamma-Ray Detection", IEEE Transactions on Nuclear Science, Vol. 65, No. 8, pp. 1-8, 2018 .

[8] C. F.Yang, C. Q. Feng, S. B. Liu, and Q. An, "FPGA-Based $n / Y$ Pulse Shape Discrimination for BaF2 Detector using 2-GSPS Fast Waveform Sampling", NUCL SCI TECH, Vol. 28 , No. 2, pp. 1-6, 2017.

[9] K. Yang, P. R. Menge, and V. Ouspenski, "Li Co-Doped NaI:Tl (NaIL) A Large Volume Neutron-Gamma Scintillator with Exceptional Pulse Shape Discrimination", IEEE Transactions on Nuclear Science, Vol. 64, No. 8, pp. 2406-2413, 2017.

[10] S. Clarke, M. Flaska, S. Pozzi, and P. Peerani, "Neutron And Gamma-Ray Cross-Correlation Measurements Of Plutonium Oxide Powder", Nuclear Inst. and Methods in Physics Research Section A: Accelerators, Spectrometers, Detectors and Associated Equipment, Vol. 604, No. 3, pp. 618-623, 2009.

[11] M. Flaska and S. A. Pozzi, "Digital Pulse Shape Analysis for The Capture-Gated Liquid Scintillator BC-523a", Nuclear Inst. and Methods in Physics Research Section A: Accelerators, Spectrometers, Detectors and Associated Equipment, Vol. 599, No. (2-3), pp. 221-225, 2009

Table (8): Discrimination results using Hilbert transformation with SVM

\begin{tabular}{|c|c|c|c|c|c|c|}
\hline \multirow{2}{*}{$\begin{array}{c}\text { SNR } \\
(\mathbf{d B})\end{array}$} & \multicolumn{2}{|c|}{$\begin{array}{c}\text { Discrimination Rate } \\
(\%)\end{array}$} & \multicolumn{3}{|c|}{$\begin{array}{c}\text { Gamma } \\
\text { misclassification }\end{array}$} & \multicolumn{2}{|c|}{$\begin{array}{c}\text { Neutron } \\
\text { misclassification }\end{array}$} \\
\cline { 2 - 7 } & $\begin{array}{c}\text { Direct } \\
\text { signal }\end{array}$ & $\begin{array}{c}\text { Normalized } \\
\text { signal }\end{array}$ & $\begin{array}{c}\text { Direct } \\
\text { signal }\end{array}$ & $\begin{array}{c}\text { Normalized } \\
\text { signal }\end{array}$ & $\begin{array}{c}\text { Direct } \\
\text { signal }\end{array}$ & $\begin{array}{c}\text { Normalized } \\
\text { signal }\end{array}$ \\
\hline-20 & 62.9496 & 57.7922 & 18.2318 & 20.8666 & 18.8187 & 21.3412 \\
\hline-15 & 72.9271 & 63.2493 & 13.4241 & 16.8581 & 13.6489 & 19.8926 \\
\hline-10 & 86.1763 & 74.2882 & 6.3811 & 10.9391 & 7.4426 & 14.7727 \\
\hline-5 & 96.9281 & 86.1139 & 1.3986 & 4.6454 & 1.6733 & 9.2408 \\
\hline 0 & 99.9625 & 96.9905 & 0.0375 & 0.4745 & 0 & 2.5350 \\
\hline 5 & 100 & 99.8626 & 0 & 0 & 0 & 0.1374 \\
\hline 10 & 100 & 100 & 0 & 0 & 0 & 0 \\
\hline
\end{tabular}


[12] M. Hamel, A. M. Frelin-Labalme, V. Simic, and S. Normand, " $A$ new Fluorophore Highly Efficient for Fast Neutrons/Gamma-Rays Discrimination in Liquid Media", Nuclear Inst. and Methods in Physics Research Section A: Accelerators, Spectrometers, Detectors and Associated Equipment, Vol. 602, No. 2, pp. 425431, 2009.

[13] N. Hawkes, K. Gamage, and G. Taylor, "Digital Approaches To Field Neutron Spectrometry", Radiation Measurements, Vol. 45, No. 10, pp. 1305-1308, 2010.

[14] A. Lavagno, G. Gervino, and C. Marino, "High Efficiency Large Volume Multi-Parametric Neutron Detector", Nuclear Inst. and Methods in Physics Research Section A: Accelerators, Spectrometers, Detectors and Associated Equipment, Vol. 617, No. (1-3), pp. 492-494, 2010.

[15] S. Pozzi, S. Clarke, M. Flaska, and P. Peerani, "Pulse-Height Distributions of Neutron and Gamma Rays from Plutonium-Oxide Samples", Nuclear Inst. and Methods in Physics Research Section A: Accelerators, Spectrometers, Detectors and Associated Equipment, Vol. 608, No. 2, pp. 310-315, 2009.

[16] R. T. Schiffer, M. Flaska, S. A. Pozzi, S. Carney, and D. D. Wentzloff, "A Scalable FPGA-Based Digitizing Platform for Radiation Data Acquisition", Nuclear Inst. and Methods in Physics Research Section A: Accelerators, Spectrometers, Detectors and Associated Equipment, Vol. 652, No. 1, pp. 491-493, 2011.

[17] A. Villano, F. Becchetti, J. Kolata, M. Ojaruega, and A. Roberts, "Efficiency Measurements Of Deuterated Liquid Scintillators Using Coincidence Events", Nuclear Inst. and Methods in Physics Research Section A: Accelerators, Spectrometers, Detectors and Associated Equipment, Vol. 652, No. 1, pp. 280-283, 2011.

[18] S. Marrone, et al., "Pulse Shape Analysis of Liquid Scintillator for Neutron Studies" Nuclear Inst. and Methods in Physics Research Section A: Accelerators, Spectrometers, Detectors and associated Equipment, Vol. 490, pp. 299-307, 2002.

[19] F. Belli, B. Esposito, D. Marocco and M. Riva, "A Study on The Pulse Height Resolution Of Organic Scintillator Digitized Pulses", Fusion Engineering and Design, Vol. 88, No. (6 - 8), pp.1271$1275,2013$.

[20] M. E. Hammad, H. Kasban, R. M. Fikry, Moawad I. Dessoky, O. Zahran, S. M. S. Elaraby and F. E. Abd El-Samie, "Digital Pulse Processing Algorithm for Neutron and Gamma Rays Discrimination", Accepted for publication in Analog Integrated Circuits and Signal Processing, Springer, 2019.

[21] C. C. Funk, J. Theiler, D. A. Roberts and C. C. Borel, "Clustering To Improve Matched Filter Detection Of Weak Gas Plumes In Hyperspectral Thermal Imagery", IEEE Trans. Geosci. Remote Sens., Vol. 39, No. 7, pp. 1410-1420, 2001.

[22] H. Kasban, O. Zahran, H.Arafa, M. El-Kordy, S. M. S. Elaraby, F.E.A. El-Samie, "Support Vector Machines And Artificial Neural Networks For Identification Of Residence Time Distribution Signals", Int. Eng. J., Vol. 6, No. 3, pp. 1803-1808, 2015.

[23] H. Kasban, O. Zahran, H. Arafa, M. El-Kordy, S. M. S. Elaraby, F.E.A.El-Samie, "Welding Defect Detection From Radiographic Image Using Cepstral Approach", Nondestruct. Test. Eval. (NTD\&E), Vol. 44, No 2, pp. 226-231, 2011.

[24] O. Zahran, H. Kasban, M. El-Kordy and F. E. A.El-Samie, "Automatic Weld Defect Identification From Radiographic Images", Nondestruct. Test. Eval. (NTD\&E), Vol. 57, pp. 26-35, 2013.

[25] C. W. Hsu, C .C .Chang and C. J. Lin, "A Practical Guide To Support Vector Classification", National Taiwan Uni, Taipei 106, Taiwan, 2007. 